University of Nebraska - Lincoln

DigitalCommons@University of Nebraska - Lincoln

2005

\title{
Noncovalent and Nonspecific Molecular Interactions of Polymers with Multiwalled Carbon Nanotubes
}

\author{
Durairaj Baskaran \\ University of Tennessee - Knoxville \\ Jimmy W. Mays \\ University of Tennessee - Knoxville \\ Matthew S. Bratcher \\ U.S. Army Research Laboratory
}

Follow this and additional works at: https://digitalcommons.unl.edu/usdoepub

Part of the Bioresource and Agricultural Engineering Commons

Baskaran, Durairaj; Mays, Jimmy W.; and Bratcher, Matthew S., "Noncovalent and Nonspecific Molecular Interactions of Polymers with Multiwalled Carbon Nanotubes" (2005). US Department of Energy Publications. 31.

https://digitalcommons.unl.edu/usdoepub/31

This Article is brought to you for free and open access by the U.S. Department of Energy at DigitalCommons@University of Nebraska - Lincoln. It has been accepted for inclusion in US Department of Energy Publications by an authorized administrator of DigitalCommons@University of Nebraska - Lincoln. 


\title{
Noncovalent and Nonspecific Molecular Interactions of Polymers with Multiwalled Carbon Nanotubes
}

\author{
Durairaj Baskaran, ${ }^{*}, \dagger$ Jimmy W. Mays, ${ }^{\dagger, \dagger}$ and Matthew S. Bratcher ${ }^{\S}$ \\ Department of Chemistry, University of Tennessee, 552 Buehler Hall, Knoxville, Tennessee 37996, \\ Chemical Sciences Division, Oak Ridge National Laboratory, Oak Ridge, Tennessee 37831, \\ and U.S. Army Research Laboratory, Weapons and Materials Research Directorate, AMSRL-WM-MA, \\ $A P G$, Maryland 21005
}

Received December 7, 2004. Revised Manuscript Received March 14, 2005

\begin{abstract}
Polymer composites containing variable amounts of multiwalled carbon nanotubes (MWNTs) have been prepared using solution dispersion and melt-shear mixing. Various polymer composites with $1 \mathrm{wt}$ $\%$ MWNTs have been found to dissolve homogeneously in organic solvents. The amount of polymer coated or wrapped MWNTs dissolved in the solution was quantified using UV-vis absorbance at 500 $\mathrm{nm}$ and the concentration ratio of [MWNTs $]_{\text {solution }} /[\mathrm{MWNTs}]_{\text {composite }}$ was close to 1 . A nonspecific polymer adsorption through multiple-weak molecular interactions of $\mathrm{CH}$ groups with MWNTs in the composites has been identified through FTIR spectroscopy. The composites of polybutadiene with different wt $\%$ of MWNTs showed slight changes in the $\mathrm{CH}$ bending vibrations, indicating the presence of intermolecular $\mathrm{CH}-\pi$ interactions. The dissolution of various polymer composites containing low concentration of MWNTs in organic solvents was attributed to polymer coating on the MWNTs via noncovalent and nonspecific $\mathrm{CH}-\pi$ interactions. The dissolution of MWNTs in organic solvents using common polymers used in this study indicates that the coating or wrapping is a general phenomenon occurring between polymers and carbon nanotubes.
\end{abstract}

\section{Introduction}

Carbon nanotubes (CNTs) are the most promising candidate for use as nanofibers in designing novel ultrahigh strength polymer composites. ${ }^{1-3}$ It is believed that the exotic mechanical and electrical properties of the CNTs will enhance the overall performance of polymer-carbon nanotube composites as well as open up new applications for such composites. ${ }^{4}$ A good interfacial adhesion between the CNTs and the polymer is essential for an efficient load transfer in composite applications. A stumbling block in developing polymer-carbon nanotube composites is the difficulty in dispersing the CNTs and controlling their orientation. The advantages of carbon nanotube fillers have not been fully realized so far due to the difficulty in obtaining welldispersed nanocomposites.

Funtionalization of CNTs is the strategy used to enhance dispersion in organic media. There are different methods used for the functionalization of CNTs through covalent and noncovalent reactions with organic molecules including polymers. ${ }^{5-18}$ An easy and viable method to produce stable

* Corresponding author. Permanent address: Division of Polymer Science and Engineering, National Chemical Laboratory, Pune- 411 008, India. E-mail: baskaran@utk.edu.

University of Tennessee.

¥ Oak Ridge National Laboratory.

$\S$ U.S. Army Research Laboratory.

(1) Ajayan, P. M. Chem. Rev. 1999, 99, 1787-1799.

(2) Ajayan, P. M.; Zhou, O. Z. In Carbon Nanotubes, Topics Appl. Phys.; Dresselhaus, M. S., Dresselhaus, G., Avouris, P., Eds.; SpringerVerlag: Berlin, 2001; Vol. 80, pp 391-425.

(3) Frankland, S. J. V.; Caglar, A.; Brenner, D. W.; Griebel, M. J. Phys. Chem. B 2002, 106, 3046-3048.

(4) Vivekchand, S. R. C.; Sudheendra, L.; Sandeep, M.; Govindaraj, A.; Rao, C. N. R. J. Nanosci. Nanotechnol. 2002, 2, 631. carbon nanotube dispersion is noncovalent polymer wrapping using specialty polymers. Smalley and co-workers ${ }^{19}$ reported a dissolution of single-walled carbon nanotubes (SWNTs) by polymer wrapping using poly(vinyl pyrrolidone) and poly(styrene sulfonate). Stoddart and co-workers ${ }^{8}$ reported polymerwrapped SWNTs using poly(metaphenylene vinylene). However, the dissolution of CNTs using polymer wrapping has

(5) Chen, J.; Hamon, M. A.; Hu, H.; Chen, Y.; Rao, A. M.; Eklund, P. C.; Haddon, R. C. Science 1998, 282, 95-98.

(6) Chen, Y.; Haddon, R. C.; Fang, S.; Rao, A. M.; Eklund, P. C.; Lee, W. H.; Dickey, E. C.; Grulke, E. A.; Pendergrass, J. C.; Chavan, A.; Haley, B. E.; Smalley, R. E. J. Mater. Res. 1998, 13, 2423-2431.

(7) Holzinger, M.; Vostrowsky, O.; Hirsch, A.; Hennrich, F.; Kappes, M.; Weiss, R.; Jellen, F. Angew. Chem., Int. Ed. 2001, 40, 4002

(8) Star, A.; Stoddart, J. F.; Steuerman, D.; Diehl, M.; Boukai, A.; Wong, E. W.; Yang, X.; Chung, S.-W.; Choi, H.; Health, J. R. Angew. Chem., Int. Ed. 2001, 40, 1721-1725.

(9) Georgakilas, V.; Kordatos, K.; Prato, M.; Guldi, D. M.; Holzinger, M.; Hirsch, A. J. Am. Chem. Soc. 2002, 124, 2002.

(10) Huang, W.; Fernando, S.; Allard, L. F.; Sun, Y.-P. Nano Lett. 2003, $3,565-568$

(11) Sun, Y.-P.; Fu, K.; Lin, Y.; Huang, W. Acc. Chem. Res. 2002, 35, $1096-1104$.

(12) Lin, Y.; Rao, A. M.; Sadanadan, B.; Kenik, E. A.; Sun, Y.-P. J. Phys. Chem. B 2002, 106, 1294-1298.

(13) Hill, D. E.; Lin, Y.; Rao, A. M.; Allard, L. F.; Sun, Y.-P. Macromolecules 2002, 35, 9466-9471.

(14) Huang, W.; Taylor, S.; Fu, K.; Lin, Y.; Zhang, D.; Hanks, T. W.; Rao, A. M.; Sun, Y. Nano Lett. 2002, 2, 311-314.

(15) Baskaran, D.; Mays, J. W.; Bratcher, M. S. Angew. Chem., Int. Ed. 2004, 43, 2138-2142.

(16) Qin, S.; Qin, D.; Ford, W. T.; Resasco, D. E.; Herrera, J. E. J. Am. Chem. Soc. 2004, 126, 170-176.

(17) Saini, R. K.; Chiang, I. W.; Peng, H.; Smalley, R. E.; Billups, W. E.; Hauge, R. H.; Margrave, J. L. J. Am. Chem. Soc. 2003, 125, 36173621.

(18) Chen, R. J.; Zhang, Y.; Wang, D.; Dai, H. J. Am. Chem. Soc. 2001, $123,3838-3839$.

(19) O’Connell, M. J.; Boul, P. J.; Ericson, L. M.; Huffman, C. B.; Wang, Y.; Haroz, E. H.; Kuper, C.; Tour, J. M.; Ausman, K. D.; Smalley, R. E. Chem. Phys. Lett. 2001, 342, 265-271. 
been shown to occur only with a few specific polymers. Shvartzman-Cohen et al. recently showed dispersion of SWNTs using micelles of diblock copolymers. ${ }^{20,21}$ It is known that the CNTs are electron-rich molecules and any electron-deficient molecules can interact with them and form donor-acceptor complexes, much the same way as the electron-rich molecules form $\pi-\pi$ complexes. The $\pi-\pi$ interactions of CNTs with electron-rich molecules have been used for the noncovalent functionalization. ${ }^{18,22-26}$ Recently, Eklund, Murray, and co-workers and others have shown that the noncovalent interactions of CNTs with small molecules other than $\pi-\pi$ interactions lead to adsorption. ${ }^{22,27-29}$

Adsorption could also occur from molecular interactions between carbon-hydrogen groups ( $\mathrm{CH}$ groups) and $\pi$ systems and the existence of $\mathrm{CH}-\pi$ interactions has been known for several years. ${ }^{30-34} \mathrm{Klopman}^{35,36}$ has classified the $\mathrm{CH}-\pi$ interaction as a soft acid/soft base interaction using orbital energy data of acids and bases. Although the strength of $\mathrm{CH}-\pi$ interactions is only one-tenth of the hydrogen bond, their cooperative multiple interactions significantly influence many chemical and biochemical phenomena. ${ }^{33}$ For example, staining of cotton by dyes containing aromatic groups is a result of many such weak interactions $(\mathrm{OH}-\pi$ and $\mathrm{CH}-\pi)$. Similarly, a fine dispersion of carbon black/fiber within a matrix rubber is also due to the presence of many $\mathrm{CH}-\pi$ interactions. Thus, we reasoned that a long molecule containing many $\mathrm{CH}$ linkages would sufficiently interact with carbon nanotubes and form molecular complex under appropriate conditions. To verify this hypothesis, we used different types of polymers as $\mathrm{CH}$ donor molecules for examining the effect of $\mathrm{CH}-\pi$ interaction with the multiwalled carbon nanotubes (MWNTs) (Scheme 1). In this paper, we describe the presence of a weak noncovalent and nonspecific $\mathrm{CH}-\pi$

(20) Shvartzman-Cohen, R.; Levi-Kalisman, Y.; Nativ-Roth, E.; YerushalmiRozen, R. Langmuir 2004, 20, 6085-6088.

(21) Shvartzman-Cohen, R.; Nativ-Roth, E.; Baskaran, E.; Levi-Kalisman, Y.; Szleifer, I.; Yerushalmi-Rozen, R. J. Am. Chem. Soc. 2004, 126 , $14850-14857$.

(22) Zhang, J.; Lee, J.-K.; Wu, Y.; Murray, R. W. Nano Lett. 2003, 3, 403-407.

(23) Li, H.; Zhou, B.; Lin, Y.; Gu, L.; Wang, W.; Fernando, S.; Kumar, S.; Allard, L. F.; Sun, Y.-P. J. Am. Chem. Soc. 2004, 126, 10141015.

(24) Kondratyuk, P.; Yates, J. T., Jr. Chem. Phys. Lett. 2004, 383, 314316.

(25) Stan, G.; Bojan, M. J.; Curtarolo, S.; Gatica, S. M.; Cole, M. W. Phys. Rev. B 2000, 62, 2173.

(26) Zhao, J.; Lu, J. P.; Han, J.; Yang, C.-K. Appl. Phys. Lett. 2003, 82, 3746.

(27) Sumanasekera, G. U.; Pradhan, B. K.; Romero, H. E.; Adu, K. W.; Eklund, P. C. Phys. Rev. Lett. 2002, 89, 166801

(28) Islam, M. F.; Rojas, E.; Bergey, D. M.; Johnson, A. T.; Yodh, A. G. Nano Lett. 2003, 3, 269-273.

(29) Moore, V. C.; Strano, M. S.; Haroz, E. H.; Hauge, R. H.; Smalley, R. E. Nano Lett. 2003, 3, 1379.

(30) Kodama, Y.; Nishihata, K.; Nishio, M.; Nakagawa, N. Tetrahedron Lett. 1977, 2105.

(31) Kodama, Y.; Zushi, S.; Nishihata, K.; Nishio, Y.; Uzawa, J. J. Chem. Soc., Perkin 2 1980, 1306.

(32) Araki, S.-i.; Seki, T.; Sakakibara, K.; Hirota, M.; Kodama, Y.; Nishio, M. Tetrahedron: Asymmetry 1993, 4, 555-574.

(33) Nishio, M.; Hirota, M.; Umezawa, Y. CH/pi interaction, evidence, nature and consequences; Wiley-VCH: New York, 1998.

(34) Mulliken, R. S., Person, W. B., Eds. Molecular complexes: a lecture and reprint volume; Wiley-Interscience: New York, 1969

(35) Klopman, G. J. Am. Chem. Soc. 1968, 90, 223.

(36) Klopman, G., Ed. Chemical Reactivity and Reaction Paths; WileyInterscience: New York, 1974
Scheme 1. Illustration of the Shear-Induced Polymer Wrapping over MWNTs under Melt Stirring in a Composite Containing Small Amounts of MWNTs ${ }^{a}$

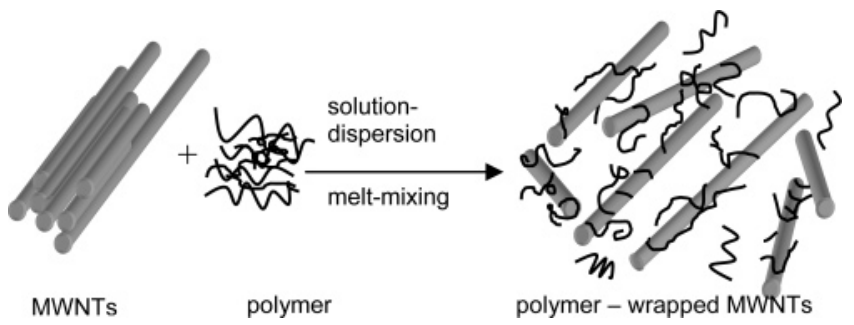

${ }^{a}$ The excess polymer is not shown for clarity.

interaction between organic molecules (both small and macro) and the MWNTs. We examine further the effect of such a noncovalent interaction in dissolution of MWNTsnanocomposites prepared with a range of polymers.

\section{Experimental Section}

Solvents such as chloroform $\left(\mathrm{CHCl}_{3}\right)$, tetrahydrofuran (THF), dichloromethane, benzene, toluene, and 1-octadecanethiol (Aldrich) were HPLC-grade solvents and used as received. Poly(ethylene oxide) dimethyl ether (PEO-Me, $M_{\mathrm{n}}=2000 \mathrm{~g} / \mathrm{mol}, M_{\mathrm{w}} / M_{\mathrm{n}}=1.11$ ), and poly(methyl methacrylate) (PMMA, $M_{\mathrm{n}}=120000 \mathrm{~g} / \mathrm{mol}, M_{\mathrm{w}} /$ $M_{\mathrm{n}}=2$ ) were obtained from Aldrich. The polymers such as polybutadiene (PBD, $\left.M_{\mathrm{n}}=120000 \mathrm{~g} / \mathrm{mol}, M_{\mathrm{w}} / M_{\mathrm{n}}=1.04\right)$, polystyrene $\left(\mathrm{PS}-\mathrm{H}, M_{\mathrm{n}}=13500 \mathrm{~g} / \mathrm{mol}, M_{\mathrm{w}} / M_{\mathrm{n}}=1.06\right)$, and polyisoprenes (PI, $M_{\mathrm{n}}=1500 \mathrm{~g} / \mathrm{mol}, 34000 \mathrm{~g} / \mathrm{mol}, 135000 \mathrm{~g} / \mathrm{mol}$, and $460000 \mathrm{~g} / \mathrm{mol}$, with $1.05<M_{\mathrm{w}} / M_{\mathrm{n}}>1.07$ ) were synthesized using standard living anionic polymerization under high vacuum. ${ }^{37}$ Poly(dimethylsiloxane) (PDMS, $M_{\mathrm{n}}=45000-60000 \mathrm{~g} / \mathrm{mol}$ ) was purchased from Gelest, Inc. (USA) and used as received.

The MWNTs were provided by NanoLab (Watertown, MA) and were used as received. The MWNTs were obtained through the chemical vapor deposition method and were purified with an HF wash to remove residual catalyst. The resulting product has approximately $1 \mathrm{~mol} \%$ carboxylic acid groups with respect to the carbon atoms of the MWNTs as determined by titration, X-ray photoelectron spectroscopy (XPS), and prompt-gamma methods. It was found that the MWNTs yielded a small quantity of iron or iron oxide particles as residue ( $\sim-5 \mathrm{wt} \%)$ in the TGA analysis.

Nanocomposite Preparation. All of the polymer composites containing various percentages of MWNTs were prepared using solution dispersion and subsequent melt-mixing procedure. A typical procedure for the preparation of a PMMA composite is given below: A solution of $5 \mathrm{~g}$ of $\mathrm{PMMA}_{120 \mathrm{~K}}$ in $50 \mathrm{~mL}$ of chloroform was added into a beaker containing $53 \mathrm{mg}$ of MWNTs in $50 \mathrm{~mL}$ of chloroform kept under sonication for $15 \mathrm{~min}$. The mixture was sonicated for an additional $15 \mathrm{~min}$ and precipitated in excess $n$-hexane. The dried, premixed composites were melt-mixed in a mini-laboratory extruder (Atlas Polymer Evaluation Products, USA) for about $15 \mathrm{~min}$ at $180{ }^{\circ} \mathrm{C}$ for PMMA and PS and at $90{ }^{\circ} \mathrm{C}$ for polybutadiene, polyisoprene, poly(dimethylsiloxane), and poly(ethylene oxide). The extruder shaft ( $\sim 1 \mathrm{~cm}$ diameter) was rotated at $60 \mathrm{rpm}$. The composite was extruded and cut into small pieces. In some case, the composite was grounded using a mortar and pestle to break it into small pieces. The actual amount of MWNTs present in the composite was determined using thermogravimetric analysis (TGA). The dissolution of the composite was accomplished by

(37) Hadjichristidis, N.; Pitsikalis, M.; Pispas, S.; Iatrou, H. Chem. Rev. 2001, 101, 3747-3792. 
A)

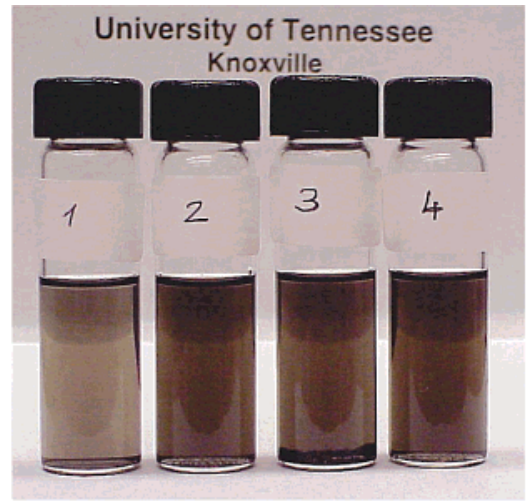

B)

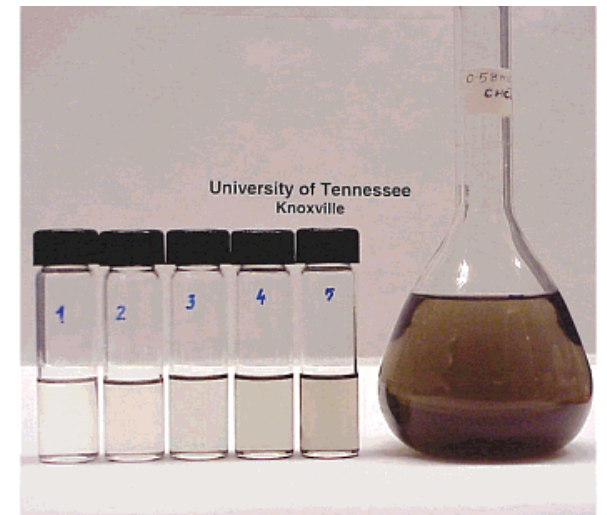

C)

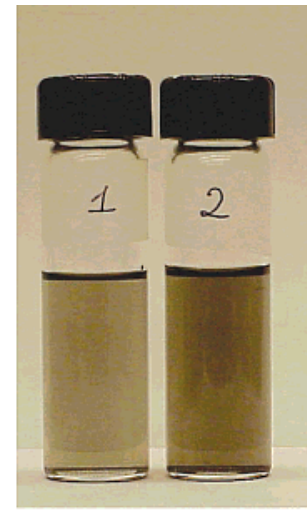

Figure 1. Various melt-mixed composite solutions of MWNTs. (A) polymer-MWNTs composite (1 wt \% MWNTs) solutions in dichloromethane photographed after 3 days, (1) PBD, (2) PIP, and (3) PS, all with [composite $]_{0}=0.2 \mathrm{mg} / \mathrm{mL}$, [MWNTs] $=0.02 \mathrm{mg} / \mathrm{mL}$, (4) PMMA, [composite $]_{0}=0.2 \mathrm{mg} / \mathrm{mL},[\mathrm{MWNTs}]$ $=0.014 \mathrm{mg} / \mathrm{mL}$. (B) PBD-MWNT composite $(0.58 \mathrm{wt} \% \mathrm{MWNTs}) \mathrm{CHCl}_{3}$ solution in standard volumetric flask, [composite $]_{0}=1 \mathrm{mg} / \mathrm{mL},[\mathrm{MWNTs}]=$ $0.0058 \mathrm{mg} / \mathrm{mL}$, photographed after 3 days and diluted to different concentrations, (1) $[\mathrm{MWNTs}]=0.0019 \mathrm{mg} / \mathrm{mL},(2)[\mathrm{MWNTs}]=0.0029 \mathrm{mg} / \mathrm{mL},(3)$ $[\mathrm{MWNTs}]=0.0035 \mathrm{mg} / \mathrm{mL}$, (4) $[\mathrm{MWNTs}]=0.0046 \mathrm{mg} / \mathrm{mL}$, and (5) $[\mathrm{MWNTs}]=0.0058 \mathrm{mg} / \mathrm{mL}$. (C) $\mathrm{C}_{18} \mathrm{H}_{37} \mathrm{SH}-\mathrm{MWTNs}$ composite (1.56 wt $\%$ MWNTs) photographed after $24 \mathrm{~h},(1)$ in chloroform, [composite $]_{0}=0.3 \mathrm{mg} / \mathrm{mL}$, [MWNTs] $=0.0047 \mathrm{mg} / \mathrm{mL}$, and (2) in THF, [composite $]_{0}=0.52 \mathrm{mg} / \mathrm{mL},[\mathrm{MWNTs}]$ $=0.0081 \mathrm{mg} / \mathrm{mL}$.

adding appropriate solvent into a known amount of composite and sonicating for $1-2 \mathrm{~min}$.

Characterization. Thermogravimetric analysis was performed using a TA Instruments TGA 2050 analyzer in air from 30 to 900 ${ }^{\circ} \mathrm{C}\left(10{ }^{\circ} \mathrm{C} / \mathrm{min}\right)$. UV-vis spectra were recorded using Thermo Spectronic's Biomate 5 instrument using dilute solutions of MWNTs and the composite dispersions. FT-IR spectra were recorded using a Bio-Red Win-IR Pro instrument with a resolution of $2 \mathrm{~cm}^{-1} . \mathrm{KBr}$ was used to prepare sample pellets. A tabletop ultrasonic cleaner (FS-20H, Fisher Scientific) operating at $40 \mathrm{kHz}$ with $80 \mathrm{~W}$ ultrasonic power was used. Polymers were characterized using gel permeation chromatography (GPC) equipped with an isocratic pump (Knauer K-501), UV detector (Knauer UV-K2501), RI detector (Knauer RI-K2301), and $1 \times 100 \AA(60 \mathrm{~cm})$, linear $(60 \mathrm{~cm})$ Polymer Standard Service (PSS) SDV gel columns. PSS WinGPC software was used to acquire and analyze the chromatogram. The GPC was calibrated with a set of PS and PMMA standards (PSS, Germany).

Raman spectra were acquired using the samples in the solid state in the backscattering mode on the "microstage" of a Dilor XY Raman spectrometer (Instruments S.A., Inc., Edison, NJ). The microstage uses confocal optics; both the excitation and the emission radiation pass through the lens nearest the sample. Under the focal and alignment conditions used, this resulted in an excitation beam diameter of $\sim 1 \mathrm{~mm}$ at the sample. The monochromator employed a 600 grooves $/ \mathrm{mm}$ grating with $200 \mu \mathrm{m}$ slits, giving a band-pass of $0.5 \mathrm{~nm}$. The $514.5 \mathrm{~nm}$ line of a Coherent Innova 200 argon ion laser was used for excitation. The laser output power of $100 \mathrm{~mW}$ was attenuated by $\sim 86 \%$ when measured at the sample position. Spectra were recorded over the range of $800-2000 \mathrm{~cm}^{-1}$ for $10-$ 12 acquisitions (with 20 s/acquisition) and averaged. The spectra were smoothed with a 7 point adjacent averaging smooth function. Transmission electron microscopy (TEM) analysis was performed using a Hitachi H-800 TEM operating with an accelerating voltage of $100 \mathrm{kV}$. Samples were prepared by dropping a $\sim 5 \mu \mathrm{L}$ solution on a freshly glow discharged carbon film supported by a 400 mesh $\mathrm{Cu}$ grid. Scanning electron microscopy (SEM) analysis was performed using a LEO 1525 FE-SEM operating with an accelerating voltage of $3 \mathrm{kV}$. Samples were prepared by dropping a $\sim 5 \mu \mathrm{L}$ solution on a freshly glow discharged piece of a silicon wafer.

\section{Results and Discussion}

Dissolution of Polymer-MWNTs Composites. Various polymer-MWNTs composites with different weight percent of MWNTs were prepared by a two-stage blending procedure. A solution of polymer was mixed with MWNTs that was kept in dispersion using sonication. After the mixture was sonicated for about $15 \mathrm{~min}$, it was immediately precipitated and dried. The precipitated composite was then subjected to shear mixing at temperatures above the $T_{\mathrm{g}}$ of the respective polymer for $15 \mathrm{~min}$. The polymers used in the preparation of composites with MWNTs are (1) polybutadiene (PBD), (2) different molecular weight polyisoprenes (PI), (3) polystyrene (PS), (4) poly(methyl methacrylate) (PMMA), and (5) poly(ethylene oxide) (PEO). The effect of $\mathrm{CH} / \pi$ interaction in the composites was examined through dissolution in organic solvents. The composites were soluble in organic solvents such as THF, $\mathrm{CHCl}_{3}$, toluene, and DMF in which the corresponding polymer is soluble. Figures $1 \mathrm{~A}$ and $\mathrm{B}$ show the vials containing various polymer composites with 1 wt \% MWNTs in dichloromethane sonicated for 1-2 min. Although the solutions contain some particulates that settled at the bottom of the vial over a period of 3 days, they were homogeneous for about $24 \mathrm{~h}$. The homogeneous polymer solution contains MWNTs up to 20 $\mathrm{mg} / \mathrm{L}$ (see the following section).

Some specific polymers with ionic groups or an electronrich backbone have been shown previously to wrap around SWNTs. ${ }^{8,19}$ The wrapping has been attributed to intermolecular noncovalent adsorption of polymers with SWNTs. However, the dissolution of MWNTs in organic solvents using common polymers used in this study indicates that the coating or wrapping is a general phenomenon occurring between polymers and carbon nanotubes. It appears that the coating or wrapping is not unique only to polymeric molecules; even a small molecule such as 1-octadecanethiol $\left(\mathrm{C}_{18} \mathrm{H}_{38} \mathrm{~S}\right)$ coats MWNTs when shear-mixed at $100^{\circ} \mathrm{C}$. Figure $1 \mathrm{C}$ shows the dissolution vials of $\mathrm{C}_{18} \mathrm{H}_{38} \mathrm{~S}-\mathrm{MWNT}$ composite containing $1.56 \mathrm{wt} \%$ MWNTs at a low composite concentration. Although the solution looks homogeneous, there are some particulates seen at the bottom of the vial, indicating an inefficient coating.

The stability of these solutions at low concentration ([polymer]/[MWNTs] $\geq 100$ ) is high, probably due to the 


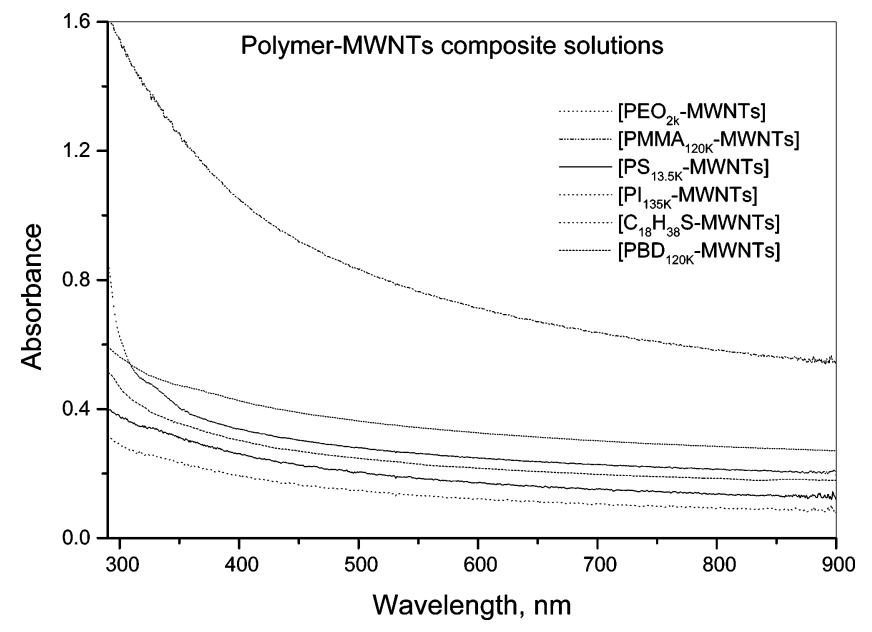

Figure 2. UV-Vis spectra of various polymer-MWNTs composites.

presence of excess polymer. The solutions do not have any visible particulates when prepared with a low concentration of MWNTs $(<20 \mathrm{mg} / \mathrm{L})$ even after several months. For example, a PBD composite (1 wt \% MWNTs) solution at a low concentration $([\mathrm{MWNTs}]=10 \mathrm{mg} / \mathrm{L})$ was stable for more than 6 months. At higher concentration of MWNTs ([polymer]/[MWNTs] $\leq 50$ ) or higher concentration of the composite ([composite] $\geq 5 \mathrm{wt} / \mathrm{v} \%$ ), the solutions were found to have a low stability and, up on aging, aggregated polymer-MWNTs bundles were seen settling to the bottom of the vial. This indicates that the polymer coverage is partial and a large surface of MWNTs is still available for van der Waals interactions in the solution. Since the solubility is an equilibrium process with an aggregated phase, the concentration of tubes and the concentration of polymer in the solution are very critical.

The polymers used here have no ionic or conjugated $\pi$-electrons to form $\pi-\pi$ intermolecular interactions with MWNTs. The coating or wrapping of the MWNTs occurs irrespective of the nature of the polymers used in this study, which indicates its nonspecificity. The carboxylic acid groups ( $1 \mathrm{~mol} \%)$ in the MWNTs do not react with polymers used in this study. Moreover, the conditions used for melt-shear mixing at a moderate speed in a min-extruder would not induce any mechano-chemical reaction between the MWNTs and the polymers. We attribute the coating or wrapping phenomenon to noncovalent and nonspecific molecular interactions between the $\mathrm{CH}$ groups of polymers and the MWNTs leading to polymer adsorption. Whether these polymer composite solutions contain suspended exfoliated individual tubes or bundles of MWNTs wrapped with polymer in the organic medium are not clear. Nonetheless, a homogeneous solution of these composites shows a strong $\mathrm{UV}-$ vis absorbance monotonically decreasing from 300 to $900 \mathrm{~nm}$ (Figure 2). The effect of noncovalent interactions is very apparent on the dissolution properties of the MWNTs, particularly, in the case of polymeric molecules than low molecular weight molecules. The adsorption is reversible and washing procedure could remove a large amount of adsorbents. However, a few percent of polymeric adsorbents could not be removed by the washing procedure, indicating the presence of a partial irreversible coating.

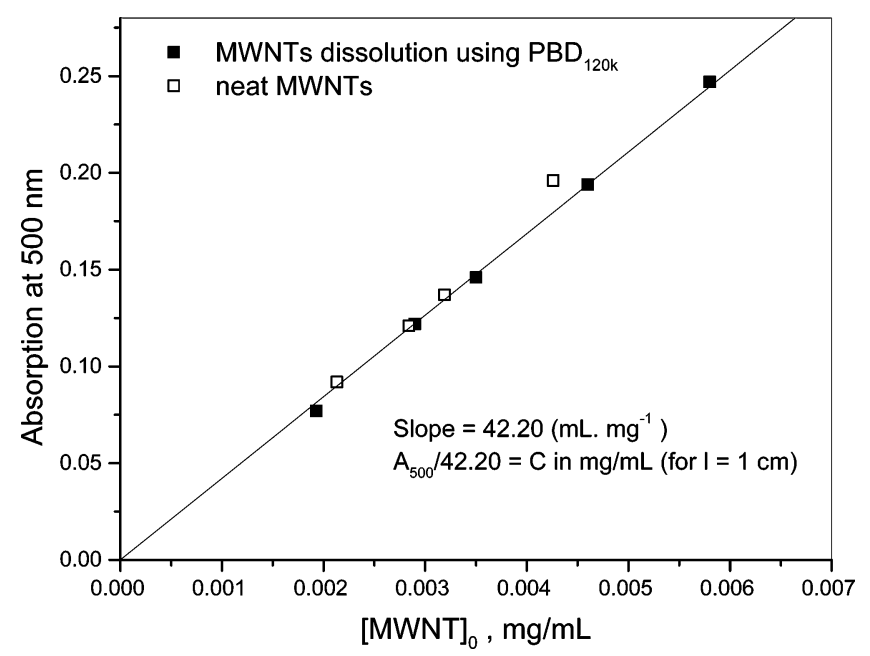

Figure 3. UV-vis spectra of PBD-MWNTs composite and neat MWNTs solutions at various concentrations in $\mathrm{CHCl}_{3}$.

Quantification of the Dissolved MWNTs. Having obtained a stable homogeneous dissolution of polymer composite at low composite and MWNT concentration ([polymer]/ $[$ MWNTs] $\geq 100$ ), we attempted to use a PBD-MWNTs composite solution (([PBD]/[MWNTs] $\geq 170)$ for the quantification of dissolved MWNTs in various composite disolutions. Accordingly, a composite was prepared using the solution dispersion melt-mixing procedure at a high ratio of polymer to MWNTs ( $0.58 \mathrm{wt} \%$ MWNTs) and dissolved in $100 \mathrm{~mL}$ of $\mathrm{CHCl}_{3}$ in a standard volumetric flask under sonication for $15 \mathrm{~min}$. A stable homogeneous MWNTs solution was obtained and no particulates were visible. From this solution different dilutions were made and the UV-vis spectra were recorded (Supporting Information, Figure S1). As the PBD has no UV-vis absorbance at and above 300 $\mathrm{nm}$, the absorbance of the composite solution above $300 \mathrm{~nm}$ is considered entirely due to the presence of dissolved MWNTs. The absorbance at $500 \mathrm{~nm}$ was plotted against the concentrations (in $\mathrm{mg} / \mathrm{mL}$ ) (Figure 3). The linear-leastsquares fit of the data passes through the origin with a slope of $42.2 \pm 0.3$, which can be treated as an apparent absorption coefficient for the batch of MWNTs used in this study. ${ }^{38}$

Dissolution of small diameter SWNTs in organic solvents was reported earlier. ${ }^{38}$ Dissolution of the MWNTs at low concentration ([MWNTs] $=4.3 \mathrm{mg} / \mathrm{L}$ ) in $\mathrm{CHCl}_{3}$ was attempted in order to verify the determined apparent absorption coefficient. It was found that it is possible to suspend the pristine MWNTs homogeneously in $\mathrm{CHCl}_{3}$ for a short time ( $\sim 15 \mathrm{~min}$ ) using sonication (30 $\mathrm{min})$. The absorbance at $500 \mathrm{~nm}$ of the neat MWNTs solution at different concentrations in $\mathrm{CHCl}_{3}$ gave a slope of $44.2 \pm 0.9$, which is close to the one determined in the presence of excess PBD (Figure 3). Thus, the slope value obtained in the presence of excess PBD can be used as an apparent absorption coefficient of the MWNTs to determine the actual concentration of MWNTs present in various composite solutions.

The concentration of MWNTs in the composite solutions was determined using an absorption intensity at $500 \mathrm{~nm}$. The results are shown in Table 2. The solutions of PBD

(38) Bahr, J. L.; Mickelson, E. T.; Bronikowski, M. J.; Smalley, R. E.; Tour, J. M. Chem. Commun. 2001, 193-194. 
Table 1. Dissolution of Various Polymer Composites Containing MWNTs in $\mathrm{CHCl}_{3}$

\begin{tabular}{|c|c|c|c|c|c|c|}
\hline \multirow[b]{2}{*}{ entry } & \multirow[b]{2}{*}{ composites $^{a}$} & \multirow[b]{2}{*}{$\begin{array}{c}([\mathrm{MWNT} s] / \\
{[\text { Polymer] }) \times 100} \\
(\mathrm{wt} \%)^{b}\end{array}$} & \multirow[b]{2}{*}{$\begin{array}{l}\text { temp } \\
\left({ }^{\circ} \mathrm{C}\right)^{c}\end{array}$} & \multicolumn{3}{|c|}{ MWNTs solution in $\mathrm{CHCl}_{3}$} \\
\hline & & & & $\begin{array}{c}\text { [MWNTs] }]_{\text {composite }} \\
\text { in dispersion } \\
(\mathrm{mg} / \mathrm{L})^{d}\end{array}$ & $\begin{array}{l}\text { Absorbance Uv } 500 \mathrm{~nm} / \\
{[\mathrm{MWNTs}]_{\text {composite }}} \\
\left(\mathrm{mL} / \mathrm{mg}^{*} \mathrm{~cm}^{-1}\right)^{e}\end{array}$ & $\begin{array}{l}{[\mathrm{MWNTs}]_{\text {solution }} /} \\
{[\mathrm{MWNTs}]_{\text {composite }}}\end{array}$ \\
\hline 1 & none & & & & 44.2 & \\
\hline 2 & polybutadiene & 0.58 & 95 & 0.058 & 42.2 & \\
\hline 3 & polybutadiene & 0.90 & 95 & 2.2 & 53.0 & 1.00 \\
\hline 4 & polybutadiene & 3.60 & 95 & 3.9 & 58.0 & 1.25 \\
\hline 5 & polybutadiene & 8.40 & 95 & 9.2 & 40.0 & 1.03 \\
\hline 6 & polyisoprene & 0.97 & 95 & 4.2 & 35.4 & 0.83 \\
\hline 7 & PMMA & 1.00 & 180 & 24.0 & 34.6 & 0.83 \\
\hline 8 & PMMA & 1.00 & 180 & 1.4 & 41.0 & 0.93 \\
\hline 9 & polystyrene & 1.00 & 180 & 5.3 & 38.5 & 0.91 \\
\hline 10 & poly(ethylene oxide) & 2.40 & 80 & 6.7 & 46.6 & 0.99 \\
\hline 11 & 1-octadecenethiol & 1.50 & 60 & 9.8 & 28.6 & 0.67 \\
\hline
\end{tabular}

${ }^{a}$ Composites prepared by solution dispersion/melt shear mixing. ${ }^{b} \mathrm{wt} \%$ of MWNTs present in the composites determined by TGA. ${ }^{c}$ Temperature at which melt shear mixing done. ${ }^{d}$ Concentration of MWNTs supposed to be in the solution on the basis of solid [MWNTs] $]_{\text {composite }}{ }^{e}$ Apparent absorption coefficient of MWNTs in various solutions.

composites prepared with different weight percentages of MWNTs from 0.58 to 8.4 wt \% show UV absorbance equivalent to the actual amount of MWNTs present in the solid composite. The ratio of absorbance at $500 \mathrm{~nm}$ to [MWNTs $]_{\text {composite }}$ for the solutions of various polymer composites containing different wt \% of MWNTs is close to the apparent absorption coefficient of MWNTs within the experimental error. The determination of the concentration ratio of $[\mathrm{MWNTs}]_{\text {solution }} /[\mathrm{MWNTs}]_{\text {composite }}$ is around 1 for several composite solutions, indicating a complete dissolution of the MWNTs (Table 1). It should be noted that the calculation is based on the assumption that the composite is homogeneous in nature. However, this way of calculation has limitations with respect to mixing efficiency. Hence, in some cases the $[\mathrm{MWNTs}]_{\text {solution }} /[\mathrm{MWNTs}]_{\text {composite }}$ varies $\pm 20-30 \%$ (Table 1 , entries 4 and 7 ).

The actual concentration of MWNTs present in the solution is plotted in Figure 4A against the calculated amount of MWNTs on the basis of the solid composite for various solutions. The concentrations of MWNTs in the solutions are close to the theoretical line, indicating an efficient dissolution of MWNTs by polymer coating. The effect of $\mathrm{CH}$ groups on the adsorption can be clearly seen from the PDMS-MWNTs composite dissolution, which deviates significantly, indicating only a low amount of MWNTs could be dissolved by PDMS (Figure 4A). This is attributed to the fact that the PDMS has a low concentration of $\mathrm{CH}$ linkages as compared to other polymers. Although the composite made out of low molecular weight molecule such as $\mathrm{C}_{18} \mathrm{H}_{38} \mathrm{~S}$ with MWNTs shows a significant deviation from theoretical line, the fact that adsorption of small molecules causes the dissolution of MWNTs in appreciable amounts is remarkable.

To demonstrate the effect of polymer's molecular weight on the dissolution properties of MWNTs, three polyisoprene-MWNTs composites using polyisoprene with different molecular weights $(1500 \mathrm{~g} / \mathrm{mol}, 34000 \mathrm{~g} / \mathrm{mol}$, and $460000 \mathrm{~g} / \mathrm{mol}$ ) were prepared. The concentration of MWNTs in these composites was $70 \mathrm{wt} \%$ in order to see the effect of the molecular weight of the polymer. Then, a constant amount of these composites was dissolved in $10 \mathrm{~mL}$ of $\mathrm{CHCl}_{3}$ and the extractable amount of dissolved MWNTs was measured using UV. As the tube's concentration in these composites is very high, the polymer $(30 \%)$ could only take as much as it can coat the tubes and bring into the dissolution. The dissolution vials were kept standing for about $9 \mathrm{~h}$ and insoluble MWNTs aggregates were found to settle at the bottom of the vial. Only the supernatant layer was taken using a pipet and analyzed for UV-vis absorbance after filtering with a thin layer of glass wool. In this way, qualitatively the amount of MWNTs present in the solution can be correlated to the molecular weight of polyisoprenes. The results indicate that the UV absorbance of these solutions at constant composite concentrations increase with increasing molecular weight of the polyisoprene in the composites (Figure 4B). The percentage of MWNTs present in the solution was calculated on the basis of the solid composite weight and the absorbance at $500 \mathrm{~nm}$ using the apparent absorption coefficient of MWNTs. A plot of \% of MWNTs vs $\log M_{\mathrm{n}}$ of polyisoprene also supports the effect of chain length on $\mathrm{CH}-\pi$ interaction (Figure $4 \mathrm{~B}$, inset).

IR and Raman Frequency Changes in the Composite. In an attempt to confirm the presence of $\mathrm{CH}-\pi$ interaction, the composite mixtures were subjected to FT-IR analysis. The presence of interactions of $\mathrm{CH}$ groups in the composite would shift the frequency of the $\mathrm{CH}$ stretching vibration of the polymer. The composites contain usually very low amounts of MWNTs $(\sim 1$ wt $\%)$. It is expected that the detection of $\mathrm{CH}$ frequencies that are interacting with MWNTs would be difficult in the presence of a huge noninteracting $\mathrm{CH}$ absorbance of the bulk polymer. Nevertheless, the PBD composites prepared with different concentrations of MWNTs show minute IR signal splitting and shifting in certain regions of the spectrum (Figure 5A). The spectrum was recorded with $2 \mathrm{~cm}^{-1}$ sensitivity and hence the average frequency shift $>2 \mathrm{~cm}^{-1}$ of any functional groups are considered important.

In general, the presence of MWNTs has broadened and shifted the $\mathrm{CH}_{\mathrm{s}}$ (stretching) and the $\mathrm{CH}_{\mathrm{b}}$ (bending) signals in the composites. The small differences in the IR frequencies observed in the composites are shown in Table 2. The $\mathrm{CH}_{b}$ vibration of the PBD in composites containing MWNTs shifted significantly $\left(-20<\Delta v \mathrm{CH}_{b}>+19\right)$ and $\mathrm{CH}_{\mathrm{s}}$ vibration had only a slight shift $\left(\Delta \nu \mathrm{CH}_{\mathrm{s}},+2\right)$ as compared to a neat PBD. The $\mathrm{CH}_{b}$ frequency of a neat PBD at 1441 $\mathrm{cm}^{-1}$ has a broad peak with a small hump (1459 $\mathrm{cm}^{-1}$ ), which splits into two doublets with main peak frequency shifts +2 and $-6 \mathrm{~cm}^{-1}$, respectively, in the presence of 13 

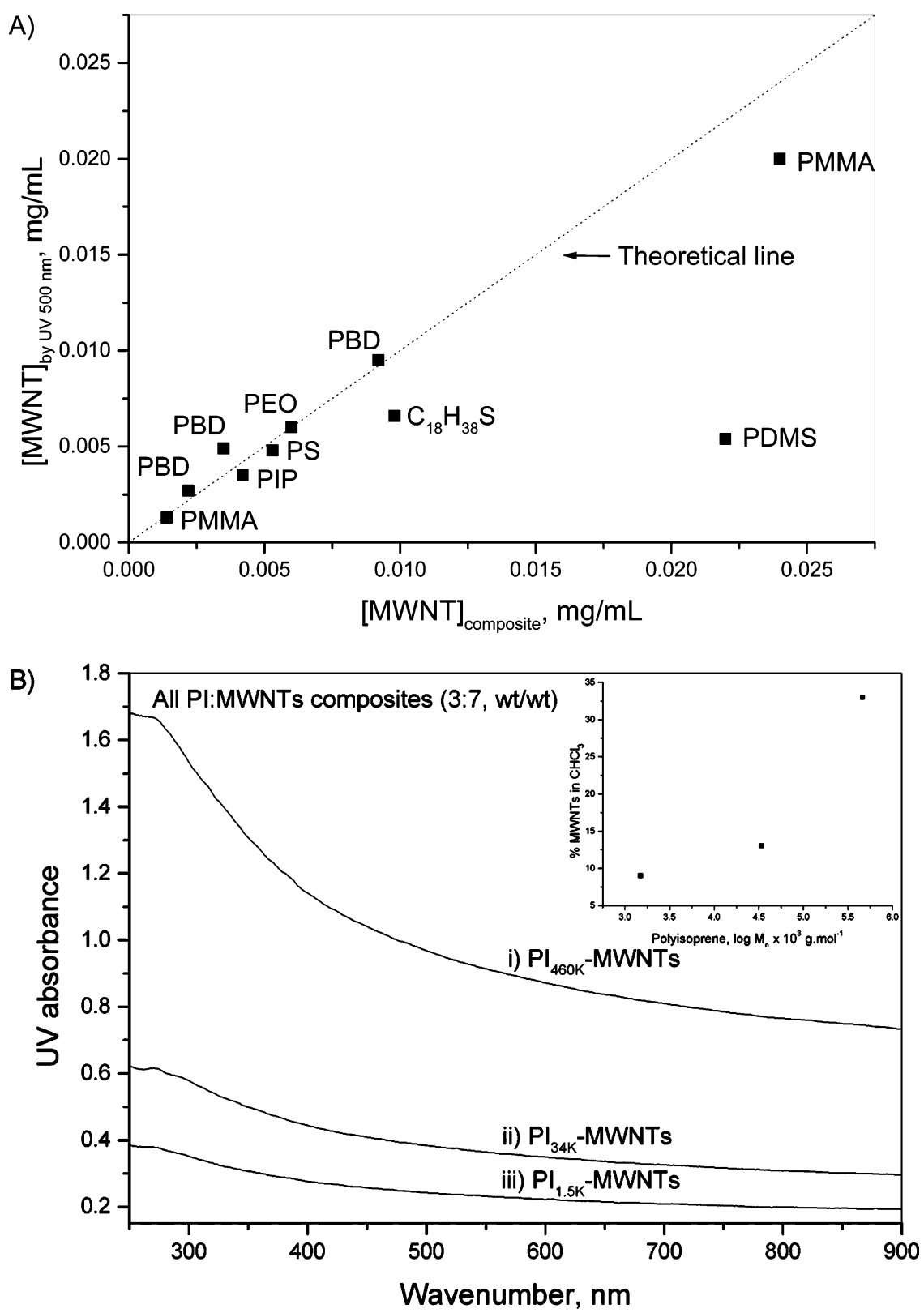

Figure 4. A) A plot of the concentration of MWNTs determined by UV absorbance at $500 \mathrm{~nm}$ versus theoretical MWNTs calculated from the composite ratio and B) UV absorbance of different molecular weight polyisoprene-MWNTs composites (all with $70 \mathrm{wt} \%$ MWNTs) solution showing increased amount of MWNTs in the supernatant solution with increasing molecular weight of the polyisoprene. The inset shows \% of MWNTs vs log Mn.

wt \% MWNTs. Upon increasing the amount of MWNTs to 63 wt $\%$, the same $\mathrm{CH}_{b}$ frequency splits wider and shifts to +4 and $-10 \mathrm{~cm}^{-1}$, indicating enhanced $\mathrm{CH}$ interactions with increasing amounts of MWNTs (Figure 5A). The signal corresponding to the interacting $\mathrm{CH}$ groups appears to be very low in intensity and separate signals could not be identified from the overwhelming presence of noninteracting $\mathrm{CH}$ groups in the system. Similarly, the changes in the absorbance of $\nu \mathrm{CH}_{\mathrm{s}}$ and $\nu \mathrm{CH}_{\mathrm{b}}$ were also noticed in the case of PMMA-MWNTs composite (Table 2). The FT-IR spectrum of a neat 1-octadecanethiol $\left(\mathrm{C}_{18} \mathrm{H}_{38} \mathrm{~S}\right)$ shows two doublets absorbance for $v \mathrm{CH}_{\mathrm{b}}$ at $1474 / 1462 \mathrm{~cm}^{-1}$ and $731 /$ $719 \mathrm{~cm}^{-1}$. In the presence of MWNTs, these signals merge into a single peak with a small hump (Supporting Information, Figure S2).

The Raman spectra of polybutadiene composites prepared with different percentages of MWNTs show shifts in tangential vibration mode peaks, G-band and D-band, to higher wavenumber with increasing concentration of PBD (Figure 5B and Supporting Information, Figure S4). Both the $\mathrm{G}$ and $\mathrm{D}$ bands of MWNTs in the composites are very broad and shifted $\sim 10$ and $\sim 15 \mathrm{~cm}^{-1}$, respectively, compared to the neat MWNTs. The higher concentration of PBD in the composite increases the probability of enhanced coverage of MWNTs surface with polymer, which affects the vibration movements of $\mathrm{C}-\mathrm{C}$ bands on the graphene plane due to $\mathrm{CH}-\pi$ interactions. The extent of vibration frequency shifting in these composites should depend on the proportions of different types of $\mathrm{CH}$ interactions between polymer molecules and the MWNTs. In a composite mixture, the molecular interactions associated with different vibration modes are heterogeneous in nature and hence overall average frequency changes are attributed to the total effect of $\mathrm{CH}-\pi$ interactions. 

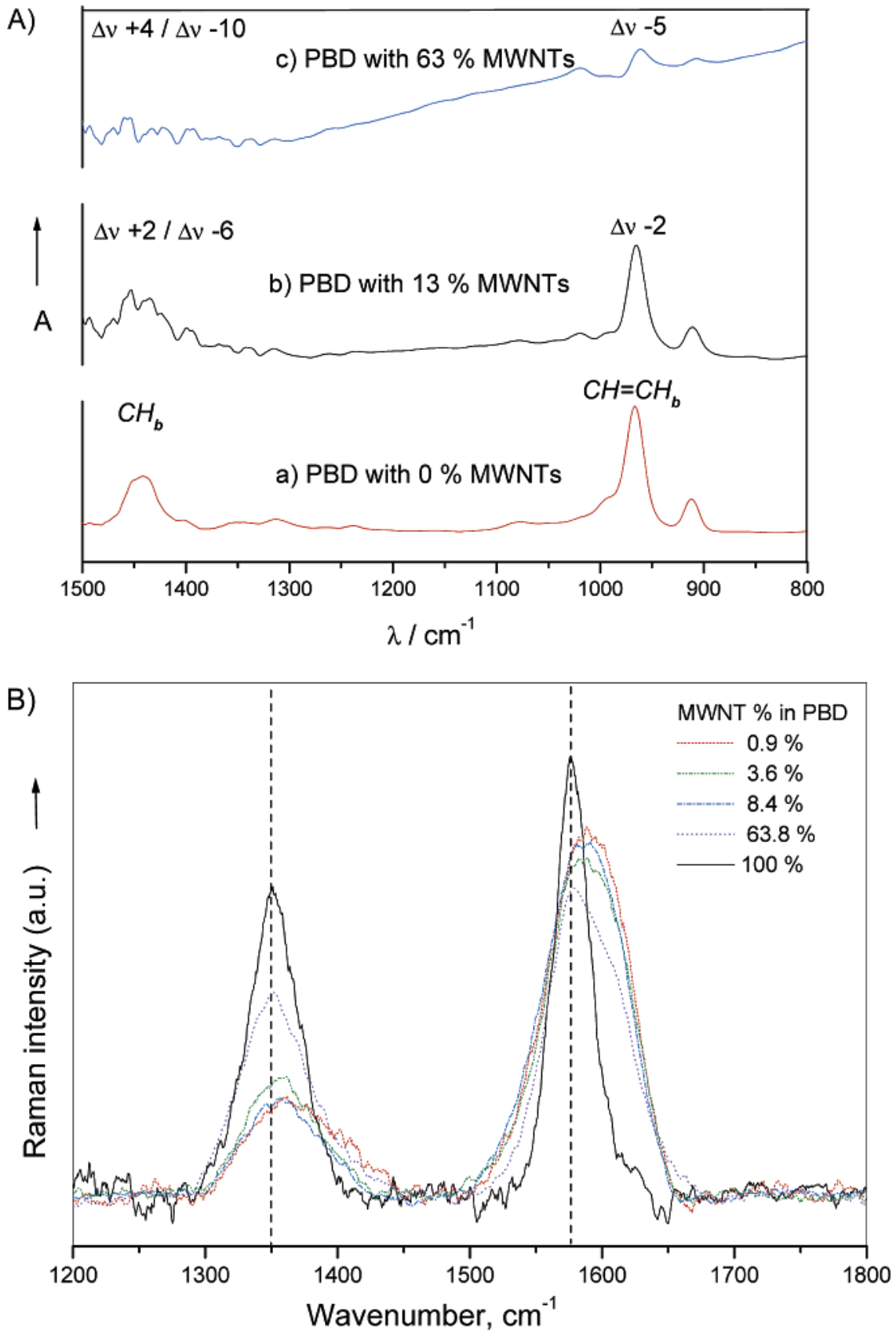

Figure 5. (A) FTIR spectra of neat PBD and their MWNTs composites and (B) Raman spectra of the tangential vibration modes of neat MWNTs and their PBD composite at $514 \mathrm{~nm}$ laser excitation.

Table 2. Frequency Shifts of $\mathrm{CH}$ Stretching and Bending Vibrations $(\Delta v)$ in Various Nanocomposite Mixtures

\begin{tabular}{|c|c|c|c|c|}
\hline nanocomposite $^{a}$ & wt $\%$ of MWNTs ${ }^{b}$ & $v$ or $\Delta v \mathrm{CH}_{\mathrm{s}}, \mathrm{cm}^{-1} c$ & $v$ or $\Delta v \mathrm{CH}_{\mathrm{b}}, \mathrm{cm}^{-1} c$ & \\
\hline PBD & 0 & $3007 / 2918 / 2845$ & $1450 / 1440$ & 966 \\
\hline PBD-MWNTs & 13.5 & $-2 / 0 / 0$ & $+19,+2 /-6,-23$ & -2 \\
\hline PBD-MWNTs & 63.8 & $-2 /+2 /+2$ & $+19,+4 /-10,-23$ & -5 \\
\hline PMMA & 0 & $2295 / 2951 / 2843$ & $1485 / 1448 / 1435$ & 910 \\
\hline PMMA-MWNTs & 0.93 & $+2 /+2,-20 /+8$ & $+3,-12 /+8 /+13,+2$ & +4 \\
\hline $\mathrm{C}_{18} \mathrm{H}_{38} \mathrm{~S}$ & 0 & $2955 / 2916 / 2851$ & $1474 / 1462$ & $731 / 719$ \\
\hline $\mathrm{C}_{18} \mathrm{H}_{38} \mathrm{~S}-\mathrm{MWNTs}$ & 1.5 & $0 /+2 /+2$ & -6 & 0 \\
\hline
\end{tabular}

${ }^{a}$ Polymer or small molecules melt-mixed with MWNTs. ${ }^{b}$ Amount of MWNTs mixed with polymer. ${ }^{c}$ Changes in the vibration frequencies of the composites against the neat polymer.

The effect of $\mathrm{CH}-\pi$ interaction is seen more significantly in the bending vibrations than in the stretching vibrations of the composite mixture. The effect of weak interactions is seen primarily on low-energy $\mathrm{CH}_{\mathrm{b}}$ vibrations rather than at high-energy $\mathrm{CH}_{\mathrm{s}}$ vibrations. The changes in the vibration frequencies in some cases are minimal. Nevertheless, the dissolution of MWNTs in organic solvents by simply meltmixing with nonfunctional polymeric or small molecules suggests that their presence is obvious. The presence of polymer coating is clearly evident from the TEM and SEM images of the composite solutions (Figure 6). A typical SEM image of the composite in solid state and in solution shows 
A)

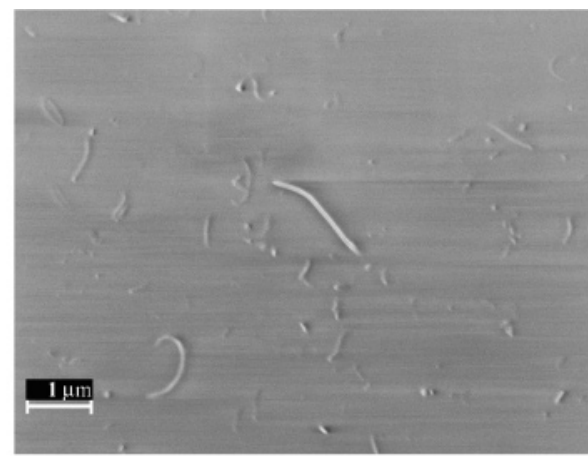

B)

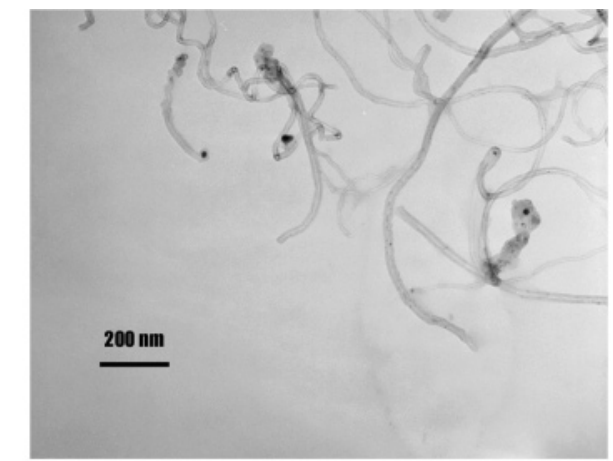

C)

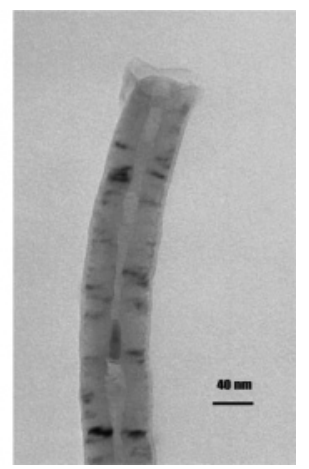

Figure 6. SEM (A) and TEM (B and C) images of MWNTs dispersed composites. (A) Polyisoprene-MWNTs (1 wt \%) prepared in dichloromethane, (B) polybutadiene-MWNTs (3.6 wt \%) dissolved in chloroform, and (C) TEM image of poly(methyl methacrylate)-MWNTs (1 wt \%) dissolved in chloroform.

A)

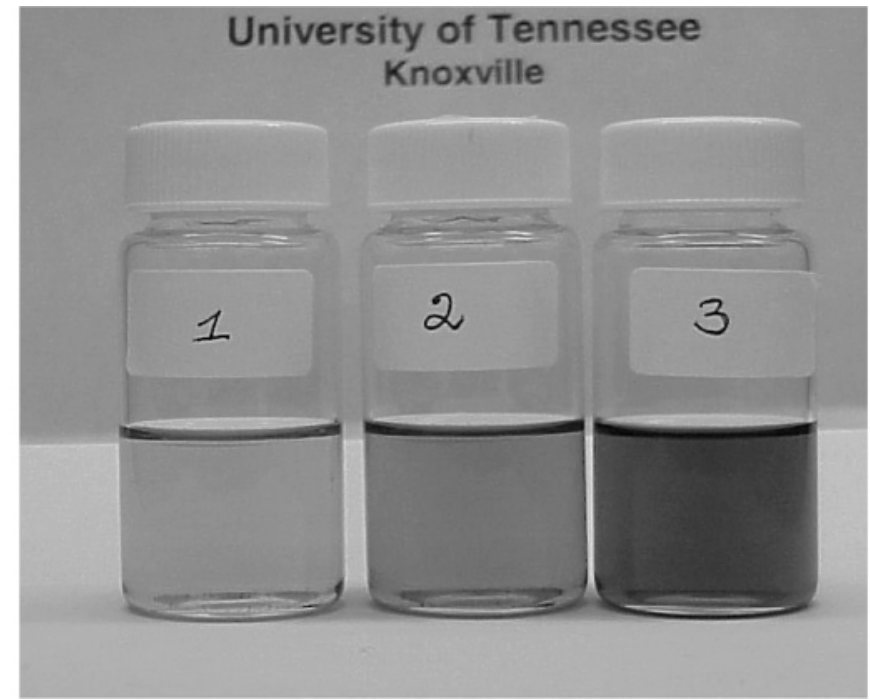

B)

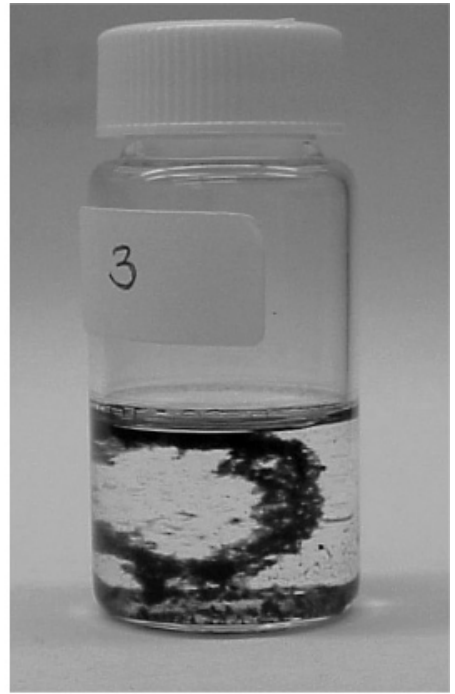

Figure 7. Dissolution vials of $\mathrm{PBD}_{120 \mathrm{k}}-\mathrm{MWNTs}$ composite solutions in $\mathrm{CHCl}_{3}$ (Table 1, runs 3-5). (A) Photographed after 10 days, (1) [composite $]_{0}=$ $0.24 \mathrm{mg} / \mathrm{mL},\left[\right.$ MWNTs] $=0.0022 ;(2)[\text { composite }]_{0}=0.11 \mathrm{mg} / \mathrm{mL},[$ MWNTs $]=0.0039 \mathrm{mg} / \mathrm{mL} ;$ (3) $[\text { composite }]_{0}=0.10 \mathrm{mg} / \mathrm{mL},[\mathrm{MWNTs}]=0.0084$ $\mathrm{mg} / \mathrm{mL}$. (B) Vial \# 3 after being aged for 24 months showing phase-separated PBD-coated MWNTs.

dispersed MWNTs present in the polymer matrix. The coverage of an amorphous polymer layer around the MWNTs can be seen in the TEM images (Figures 6 B,C) (Supporting Information, Figure S3).

It is important to mix polymer with MWNTs under a good shearing condition above the glass-transition temperature $\left(T_{\mathrm{g}}\right)$ in the absence of solvent. A simple solution mixed composite shows separation of MWNTs as soon as placed in an organic solvent. The magnitude of $\mathrm{CH}-\pi$ interactions is related to both the orbital distance and the orientation of adsorbent and carbon nanotubes. ${ }^{32}$ Hence, the reaction condition such as melt blending or shear mixing would greatly enhance the formation of $\mathrm{CH}-\pi$ interactions. In such a condition, an initial contact of a chain with the MWNTs' surface forms $\mathrm{CH}-\pi$ interaction and successive $\mathrm{CH}$ groups gradually attach with the surface, leading to complete or partial chain adsorption. In solution such an event may take place to a lesser extent due to a high chain relaxation. ${ }^{39}$ However, the probability for a maximum number of $\mathrm{CH}$ groups contact is high under shear in the absence of solvent, leading to adsorption. The loss of entropy in the adsorption process would restrict sequential adsorption of monomeric units along the chain, leaving a part of the chain segment entangled with

(39) O'Schughnessy, B.; Vavylonis, D. The Ero. Phys. J. E 2003, 11, $213-$ 230. the bulk polymer. Smalley and co-workers ${ }^{19}$ have proposed a phenomenon of reversible uniform helical wrapping in the case of poly(vinyl pyrrolidone) and SWNTs mixtures in solution. It appears that the process of polymer wrapping through $\mathrm{CH}-\pi$ interactions is not continuous and a portion of polymer likely dangles away from the surface entangled with unwrapped free polymer. The surface area of the MWNTs is quite large and a portion of the surface, which is not having intimate polymer coverage, can interact with other tubes in solution at higher concentration and slowly aggregate. In dilute solution, the probability of such an exposed surface interaction with one another is reduced and hence the equilibrium aggregation is also reduced, resulting in high stability for composite solution. The stability of the homogeneous composite solution is measured in terms of how long the MWNTs stay in a dispersed state. Figure 7A shows dissolution vials of three different $\mathrm{PBD}_{120 \mathrm{~K}}-\mathrm{MWNTs}$ composites with MWNTs concentrations of $2.2 \mathrm{mg} / \mathrm{L}, 3.9$ $\mathrm{mg} / \mathrm{L}$, and $8.4 \mathrm{mg} / \mathrm{L}$, respectively (Table 1 , runs $3-5$ ). The picture was taken after 10 days of the vials standing at room temperature, which showed a good stability of these solutions. However, on aging these solutions, PBD-coated MWNTs gradually aggregated over the period of 24 months and settled as a gel-like polymer-MWNT network (Figure 7B). 
It is worth mentioning here that previous reports ${ }^{5,10,11,40}$ concerning the functionalization of SWNTs and MWNTs using esterification and amidation reactions have employed extended stirring at high temperature in the absence of solvent. The results obtained herein show that under these conditions a considerable shear force induces a fine nonuniform coating of organic molecules on the surfaces of MWNTs through noncovalent molecular interactions that produces a substantial amount of soluble carbon nanotubes. The dissolution of MWNTs using PDMS or a low molecular weight substance such as $\mathrm{C}_{18} \mathrm{H}_{38} \mathrm{~S}$ was not efficient compared to the other polymers. This indicates that the efficiency of adsorption and its influence on the dissolution of MWNTs depends on the molecular weight or the number of $\mathrm{CH}$ groups available for the molecular interaction. Hence, a large number of $\mathrm{CH}$ groups are required to coat sufficiently and influence the dissolution properties of carbon nanotubes. The interaction of polymeric molecules with carbon nanotubes is strong through multiple $\mathrm{CH}-\pi$ interactions and that they cannot be removed completely using a simple solvent wash. Hence, the functionalization efficiencies reported for many grafting reactions should be reconsidered as in many previous reports the efficiency was calculated on the basis of weight differences of the soluble and the residual (insoluble) carbon nanotubes after the reaction. For example, Haddon and co-workers pointed out a discrepancy associated with the functionalization efficiency of SWNTs using octadeylamine, indicating a far higher amount of functionalization than the total concentration of the tubes' end-acid groups. ${ }^{41}$ It is certain that the noncovalent interactions discussed above play an important role in the functionalization reactions with carbon nanotubes. In the case of polymer adsorption, it is difficult to remove the adsorbed polymer entirely by simple solvent washing. This confirms that a certain extent of irreversible polymer coating occurs under melt-stirring conditions.

(40) Sun, Y.-P.; Huang, W.; Lin, Y.; Fu, K.; Kitaygorodskiy, A.; Riddle, L. A.; Yu, J. Y.; Carroll, D. L. Chem. Mater. 2001, 12, 2864-2869.

(41) Hamon, M. A.; Hu, H.; Bhowmik, P.; Niyogi, S.; Zhao, B.; Itkis, M. E.; Haddon, R. C. Chem. Phys. Lett. 2001, 347, 8-12.

\section{Conclusions}

The MWNT-polymer nanocomposites obtained from solution dispersion and melt shear-mixing dissolve homogeneously in organic solvents at low concentration. The stability of the nanocomposite dissolutions at a low concentration of MWNTs is high ([polymer]/[MWNTs] $\geq 100$ ). The dissolution of MWNTs using a shear mixing with various polymers was attributed to polymer (or small molecule) adsorption via noncovalent and nonspecific $\mathrm{CH}-\pi$ intermolecular interaction. The quantity of MWNTs present in the composite solutions was determined using UV-vis absorbance at $500 \mathrm{~nm}$. The ratio of $[\mathrm{MWNTs}]_{\text {solution }} /$ [MWNTs $]_{\text {composite }}$ was close to 1 for various polymer composites, indicating the nonspecific nature of $\mathrm{CH}-\pi$ interaction. The mechanism of intermolecular interaction and the physicochemical state of adsorbed molecules are not known well. However, the FTIR and Raman spectra of the composites show evidence for the presence of $\mathrm{CH}$ group interactions with MWNTs. Although the intermolecular $\mathrm{CH}-\pi$ interaction is weak, its effect in the dissolution of MWNTs in organic solvent is very apparent. The dissolution of MWNTs in organic solvents using common polymers used in this study indicates that the coating or wrapping is $a$ general phenomenon occurring between polymers and carbon nanotubes.

Acknowledgment. We thank Dr. Phil Britt, Oak Ridge National Laboratory, for facilitating the use of TGA and other instrumentation. The work at UT was supported by the U.S. Army (DAAD19-01-2-002). Work at ORNL was sponsored by the Division of Materials Science and Engineering, Office of Basic Energy Sciences, U.S. Department of Energy, under Contract No. DE-AC05-00OR22725 with Oak Ridge National Laboratory, managed and operated by UT-Battelle, LLC.

Supporting Information Available: UV-vis spectra of PBDMWNTs composite at different concentrations (Figure S1), FT-IR spectrum of MWNTs composites prepared with 1-octadecanethiol $\left(\mathrm{C}_{18} \mathrm{H}_{38} \mathrm{~S}\right)$ (Figure $\mathrm{S} 2$ ), TEM images of polymethyl methacrylateMWNTs composites (Figure S3), and Raman spectrum of MWNTs composite with $\mathrm{C}_{18} \mathrm{H}_{38} \mathrm{~S}$ (Figure $\mathrm{S} 4$ ). This material is available free of charge via the Internet at http://pubs.acs.org..

CM047866E 